\title{
Rheology of Inelastic Hard Spheres at Finite Density and Shear Rate
}

\author{
W. Till Kranz, ${ }^{1,2, *}$ Fabian Frahsa, ${ }^{3}$ Annette Zippelius, ${ }^{4}$ Matthias Fuchs, ${ }^{3}$ and Matthias Sperl ${ }^{1,2}$ \\ ${ }^{1}$ Institut für Materialphysik im Weltraum, Deutsches Zentrum für Luft- und Raumfahrt, 51170 Köln, Germany \\ ${ }^{2}$ Institut für Theoretische Physik, Universität zu Köln, 50937 Köln, Germany \\ ${ }^{3}$ Fachbereich Physik, Universität Konstanz, 78457 Konstanz, Germany \\ ${ }^{4}$ Institut für Theoretische Physik, Georg-August-Universität Göttingen, 37077 Göttingen, Germany
}

(Received 12 October 2017; revised manuscript received 11 July 2018; published 2 October 2018)

\begin{abstract}
Considering a granular fluid of inelastic smooth hard spheres, we discuss the conditions delineating the rheological regimes comprising Newtonian, Bagnoldian, shear thinning, and shear thickening behavior. Developing a kinetic theory, valid at finite shear rates and densities around the glass transition density, we predict the viscosity and Bagnold coefficient at practically relevant values of the control parameters. The determination of full flow curves relating the shear stress $\sigma$ to the shear rate $\dot{\gamma}$ and predictions of the yield stress complete our discussion of granular rheology derived from first principles.
\end{abstract}

DOI: 10.1103/PhysRevLett.121.148002

Predicting and understanding granular flow is desirable for safety and efficiency. Many geophysical flows from avalanches to landslides involve macroscopic particles and potentially threaten lives all around the planet [1,2]. A large fraction of the raw materials handled in industry comes in granular form [3]. With the advent of 3D printing technologies, this fraction will further increase $[4,5]$. Hence, efficient handling of granular flows promises considerable advantages like energy savings [6]. The crucial property of granular particles-namely that they are of macroscopic size-makes a theoretical description challenging [7-9]. First, dissipative collisions break time-reversal symmetry and place granular flows firmly in the realm of far-fromequilibrium physics $[10,11]$. Second, the macroscopic mass of an individual granular particle makes its thermal excitations negligible [7] and necessitates a driving force that is continuously acting on the granular assembly to keep it flowing [12-14].

At small volume fractions $\varphi \ll 1$ and infinitesimal shear rates $\dot{\gamma} \rightarrow 0$ standard procedures starting from the Boltzmann or Enskog equation predict a Newtonian rheology for (smooth) granular particles. Various approximate expressions exist for the viscosity as a function of inelasticity (often quantified by a coefficient of restitution $\varepsilon$ ) and volume fraction [15-20]. However, most natural and industrial granular flows occur at considerable volume fractions all the way up to close packing densities.

Published by the American Physical Society under the terms of the Creative Commons Attribution 4.0 International license. Further distribution of this work must maintain attribution to the author(s) and the published article's title, journal citation, and DOI.
Relevant shear rates $\dot{\gamma}$ are also often substantial compared to microscopic timescales.

Experimental as well as numerical studies have found a wealth of phenomena at finite densities and shear rates [21-26]. One of the earliest results of granular physics by Bagnold [21] — now commonly referred to as Bagnold scaling-is the observation that granular fluids do not follow a Newtonian rheology, but that the shear stress shows a quadratic dependence on the shear rate, $\sigma=B \dot{\gamma}^{2}$, instead. Phenomenologically, Bagnold scaling is a manifestation of shear thickening as the shear rate dependent viscosity $\eta(\dot{\gamma}) \equiv \sigma / \dot{\gamma} \sim \dot{\gamma}$ increases with shear rate. Theoretical predictions of the corresponding Bagnold coefficient $B$ are rare $[15,27,28]$ and for low density or low shear rate only. Later studies also show the opposite behavior, i.e., shear thinning, in granular fluids. Taken together, these observations imply that the Newtonian rheology is valid only in a limited part of the parameter space and capturing the full rheology in a single theoretical framework remains a challenge.

In this Letter we will show that, indeed, granular flows display all three regimes: Newtonian, shear thinning, and shear thickening [Fig. 1(a)]. We will derive the conditions to observe any of these behaviors. Based on this classification, we then calculate the relevant material properties, namely the Newtonian viscosity $\eta$, the Bagnold coefficient $B$, and, more generally, flow curves, i.e., $\sigma(\dot{\gamma})$, or, equivalently, $\eta(\dot{\gamma})$.

Formally, the stationary shear stress $\sigma$ at a constant finite shear rate can be expressed via a generalized Green-Kubo relation,

$$
\sigma=\frac{\dot{\gamma}}{V T} \int_{0}^{\infty} d t\left\langle\sigma_{x y} \mid \sigma_{x y}(t)\right\rangle_{\mathrm{ref}},
$$




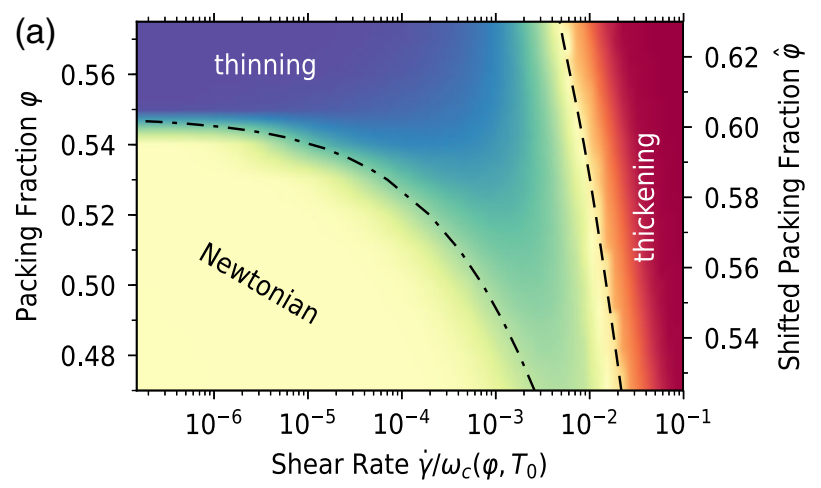

(b)
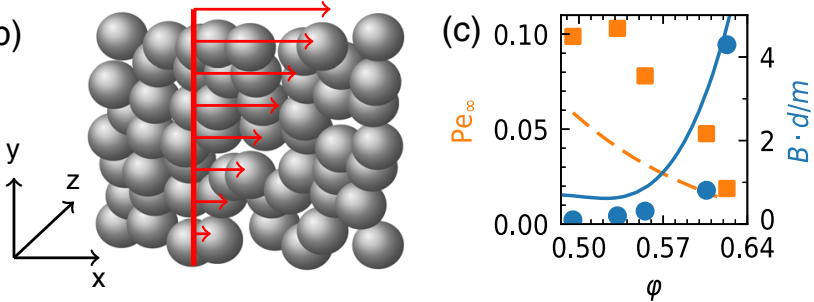

FIG. 1. (a) Theoretical dynamic state diagram of a granular fluid with coefficient of restitution $\varepsilon=0.5$ depending on packing fraction $\varphi(\hat{\varphi}:=\varphi+5.5 \%)$ and shear rate $\dot{\gamma}$. Rheological behavior $(R)$ color coded as indicated. Critical Péclet numbers $\mathrm{Pe}^{*}$ (dashed) where shear heating becomes important and $\mathrm{Pe}_{\alpha}$ (dash-dotted), where $\dot{\gamma}$ matches the intrinsic relaxation rate, $\tau_{\alpha}^{-1}$. (b) Schematic of the shear geometry with the shear profile overlayed (red). (c) Maximal Péclet number, $\mathrm{Pe}_{\infty}$ (orange dashed, left axis), and Bagnold coefficient, $B$ (blue solid, right axis), as a function of the volume fraction $\varphi$ comparing Bagnold's measurements [21] (symbols) and the kinetic theory presented here (lines).

as the time integral over the shear stress autocorrelation whose evolution depends on the flow [29]. Here $\sigma_{x y}$ is the Kirkwood shear stress defined in terms of the particle positions and momenta [30]; $V$ is the volume and $T$ the temperature of a reference state. The average $\langle\cdot\rangle_{\text {ref }}$ is performed with respect to the unsheared reference system. Although the relation was originally derived for a reference system in thermal equilibrium [29], it ultimately relates stationary states including out-of-equilibrium reference systems [31]. Our choice allows us to specify the perturbing stress in Eq. (1) microscopically. The autocorrelation function can be controlled by identifying a dominant decay channel. For thermal colloidal suspensions the slow structural relaxations close to the glass transition provides such a clearly defined, slow decay [32]. Approximating the stress autocorrelation function in Eq. (1) in terms of the density correlator $\Phi_{q}(t)$ lies at the heart of the integration through transients (ITT) formalism [29,33],

$$
\sigma \simeq \dot{\gamma} \sum_{q} \int_{0}^{\infty} d t \mathcal{V}_{\boldsymbol{q}(-t)} \Phi_{\boldsymbol{q}(-t)}^{2}(t)
$$

Here $\boldsymbol{q}(-t) \equiv(1+\mathrm{k} t) \cdot \boldsymbol{q}$ denotes the advected wave vector and the velocity gradient tensor $\mathrm{k}_{\alpha \beta}:=\dot{\gamma} \delta_{\alpha y} \delta_{\beta x}$ prescribes simple shear [Fig. 1(b)]. Note that the advected wave vector's dependence on $\dot{\gamma}$ effects a nonlinear stressshear rate relation in Eq. (2). The coupling constant $\mathcal{V}_{q q(-t)}$ can be calculated explicitly [see Refs. [31,33] and Eq. (4) below]. The ITT framework has led to a wealth of qualitative and quantitative predictions regarding the rheology of thermalized colloidal suspensions [34-37].

Power balance.-The temperature $T$ of an overdamped colloidal suspension is assumed to be controlled by a heat bath. In particular, one assumes that the work performed on the suspension by the shear force does not increase the temperature but that instead the temperature can be chosen freely. Formulating ITT for underdamped, Newtonian dynamics, a thermostat has to be included explicitly with the final results depending on the precise choice of artificial thermostat $[38,39]$. In granular flows the balance between shear heating and dissipation occurs naturally and actually controls the qualitative behavior of the granular fluid. The system we have in mind in the following is a sheared fluidized bed [40]. To be specific, let us consider a fluid composed of monodisperse smooth hard spheres of diameter $d$, and mass $m=1$, with a coefficient of normal restitution $\varepsilon$. We assume that, initially, the fluid is prepared at a given density $n$, or packing fraction $\varphi=\pi n d^{3} / 6$, and a random fluidization force is applied throughout the system with a characteristic power per particle $P_{D}$ to mimic fluidization $[41,42]$. Then the initial granular temperature $T_{0}$ results from the power balance $P_{D}=\Gamma \omega_{c}\left(T_{0}\right) T_{0}$ where $\Gamma:=\left(1-\varepsilon^{2}\right) / 3$. The collision frequency, $\omega_{c}(\varphi, T) \propto$ $\varphi \chi(\varphi) \sqrt{T}$, increases with density and temperature and $\chi(\varphi)$ denotes the pair correlation function at contact [20].

Once shear is applied with a prescribed shear rate $\dot{\gamma}$, shear heating has to be included in the power balance of the steady state,

$$
\sigma \dot{\gamma}+n P_{D}=n \Gamma \omega_{c}(T) T,
$$

resulting in a higher temperature $T>T_{0}$ (Protocol $H$ in Ref. [31]). This clearly defines two regimes: a fluidization dominated regime, where $\sigma \dot{\gamma} \ll n P_{D}$, and a shear dominated regime, where $\sigma \dot{\gamma} \gg n P_{D}$ including purely shear driven systems $\left(P_{D} \equiv 0\right)$. Choosing the particle diameter, $d$, as our length scale, and the inverse collision frequency in the stationary state, $\omega_{c}^{-1}(T)$, as our timescale, the packing

TABLE I. Definition of the critical Péclet numbers delineating the rheological regimes as functions of packing fraction $\varphi$ and coefficient of restitution $\varepsilon$. See text for details.

$$
\begin{aligned}
& \operatorname{Pe}_{\alpha}(\varphi, \epsilon)=\left(\omega_{c} \tau_{\alpha}\right)^{-1} \\
& \operatorname{Pe}^{*}(\varphi, \epsilon)=\sqrt{2} \Gamma(\varepsilon) / 2 \hat{\sigma}\left(\mathrm{Pe}^{*}, \varphi, \varepsilon\right) \\
& \operatorname{Pe}_{\infty}(\varphi, \epsilon)=\Gamma(\varepsilon) / \hat{\sigma}\left(\mathrm{Pe}_{\infty}, \varphi, \varepsilon\right)
\end{aligned}
$$


fraction, the coefficient of restitution, and the Péclet number, $\mathrm{Pe}=\dot{\gamma} / \omega_{c}(T)$, alone determine the system's state. The temperature only controls the overall timescale. The shear stress scales as $\sigma=n T \hat{\sigma}$ with a dimensionless function $\hat{\sigma}=\hat{\sigma}(\mathrm{Pe}, \varphi, \epsilon)$ because $n T$ provides the only scale for an energy density. The crossover between the regimes is expected where $\sigma \dot{\gamma} \sim n P_{D}$. This implicitly determines a critical Péclet number $\operatorname{Pe}^{*}(\varphi, \varepsilon)$ (cf. Table I).

In the fluidization dominated regime, the temperature remains independent of the shear rate such that we can linearize $\hat{\sigma} \sim \dot{\gamma}$ to find Newtonian behavior. In the shear dominated regime the power balance reads $n T \hat{\sigma} \dot{\gamma}=n T \Gamma \omega_{c}$ [43]; i.e., the collision frequency is proportional to the shear rate and the corresponding Péclet number, $\mathrm{Pe}=$ $\mathrm{Pe}_{\infty}(\varphi, \varepsilon)>0$ (cf. Table I), is independent of the shear rate. From $\dot{\gamma} \propto \omega_{c} \propto \sqrt{T}$ we obtain $T \propto \dot{\gamma}^{2}$ and, with that, $\sigma \propto T \propto \dot{\gamma}^{2}$ : Bagnold rheology is observed in the shear dominated regime where the shear rate is the only timescale and the Péclet number, $\mathrm{Pe}_{\infty}$, is fixed by the packing fraction and the coefficient of restitution. Upon increasing the shear rate $\dot{\gamma}$ towards the Bagnold regime, the temperature $T(\mathrm{Pe}) / T_{0}=\left(1-\mathrm{Pe} / \mathrm{Pe}_{\infty}\right)^{-2 / 3}$ diverges. This implies that $\mathrm{Pe}_{\infty}$ is the maximal Péclet number, which cannot be exceeded in a granular fluid.

Glassy dynamics and yield stress. - Granular fluids have been found to undergo a glass transition [14] at a critical packing fraction $\varphi_{c}(\varepsilon)$, which increases with increasing dissipation [44]. Upon approaching the (granular) glass transition, $\varphi \nearrow \varphi_{c}$, the characteristic correlation time for density fluctuations, $\tau_{\alpha}$, diverges. For $\dot{\gamma} \ll \tau_{\alpha}^{-1}$ the rheology is Newtonian, as the granular fluid can respond immediately to the slow shear deformations. However, the viscosity diverges as $\varphi \nearrow \varphi_{c}$. For higher prescribed shear rates, $\dot{\gamma} \gtrsim \tau_{\alpha}^{-1}$ [i.e., $\operatorname{Pe} \gtrsim \operatorname{Pe}_{\alpha}(\varphi, \varepsilon)$, cf. Table I], the glass is forcibly molten.

At the lowest order it can be assumed that $\Phi_{q}(t \rightarrow \infty) \propto$ $e^{-\dot{\gamma} t}$ for $\mathrm{Pe}>\mathrm{Pe}_{\alpha}$. Consequently the Green-Kubo-Integral, Eq. (2), becomes independent of the shear rate, i.e., $\sigma(\dot{\gamma}) \approx$ const. In terms of the viscosity $\eta(\dot{\gamma}) \sim 1 / \dot{\gamma}$ we expect to observe shear thinning. For even higher shear rates, $\mathrm{Pe}>\mathrm{Pe}^{*}$, shear heating will dominate and eventually bend the flow curve to the shear thickening Bagnold regime.
For densities above the glass transition, $\varphi>\varphi_{c}, \mathrm{Pe}_{\alpha} \rightarrow 0$ and the Newtonian regime vanishes altogether. Instead, a finite (dynamical) yield stress $\sigma_{y}:=\sigma(\dot{\gamma} \rightarrow 0)>0$ emerges, which has to be overcome to melt the amorphous granular glass.

Granular integration through transients.-Recently $[31,45]$, we derived a non-equilibrium ITT formalism for granular fluids (GITT) employing granular mode-coupling theory (MCT) $[44,46]$. As a central result, we obtain an expression for the generalized Green-Kubo relation in isotropic approximation,

$$
\begin{aligned}
\frac{\sigma}{n T}= & \frac{\sigma_{0}}{n T}+\operatorname{Pe} \frac{1+\varepsilon}{2 \varphi} \int_{0}^{\infty} \frac{d \tau}{\sqrt{1+(\operatorname{Pe} \tau)^{2} / 3}} \\
& \times \int_{0}^{\infty} \frac{d q^{*} q^{* 4}}{360 \pi} \times \frac{S_{q^{*}(-\tau)}^{\prime} S_{q^{*}}^{\prime}}{S_{q^{*}}^{2}} \Phi_{q^{*}(-\tau)}^{2}(\tau),
\end{aligned}
$$

extending the low-density, Enskog prediction $\sigma_{0}$ [19]. Here $S_{q}^{\prime}$ denotes the derivative of the static structure factor, $q^{*}:=q d$ and $\tau:=\omega_{c} t$, are the dimensionless wave number and time, respectively, and $q^{*}(-\tau)=q^{*} \sqrt{1+(\mathrm{Pe} \tau)^{2} / 3}$.

Numerically solving Eq. (4) together with the granular MCT equations yields flow curves like those shown in Fig. 2 as well as the critical Péclet numbers $\mathrm{Pe}_{\alpha}, \mathrm{Pe}^{*}$, and $\mathrm{Pe}_{\infty}$ [Fig. 1(a)]. For more details, see Ref. [31]. Indeed, we observe all the regimes discussed above: (i) Newtonian behavior, $\sigma=\eta \dot{\gamma}$, for low densities and shear rates, $\mathrm{Pe}<\mathrm{Pe}_{\alpha}$; (ii) a yield stress above the glass transition density, and thinning for $\mathrm{Pe}_{\alpha}<\mathrm{Pe}<\mathrm{Pe}^{*}$; and (iii) the Bagnold regime, $\sigma=B \dot{\gamma}^{2}$, for large shear rates, $\mathrm{Pe}>\mathrm{Pe}^{*}$, where the flow curves end at $\mathrm{Pe}=\mathrm{Pe}_{\infty}$ [Figs. 2(c) and 2(d)]. Considering the generalized viscosity $\eta(\dot{\gamma})$ [Figs. 3(a) and 3(b)] makes the thinning and thickening terminology particularly transparent. Note that we span many orders of magnitude in shear rate and viscosity. The critical density's dependence on $\varepsilon$ [Fig. 3(d)], $\varphi_{c}(\epsilon)$, strongly influences the flow behavior at fixed flow conditions if the coefficient of restitution is varied (cf. Figs. 2 and 3). The rheological regimes can be classified by $R:=$ $\partial \ln \eta(\dot{\gamma}) / \partial \ln \dot{\gamma}$ [color coded in Fig. 1(a)]. Here, $R=0$
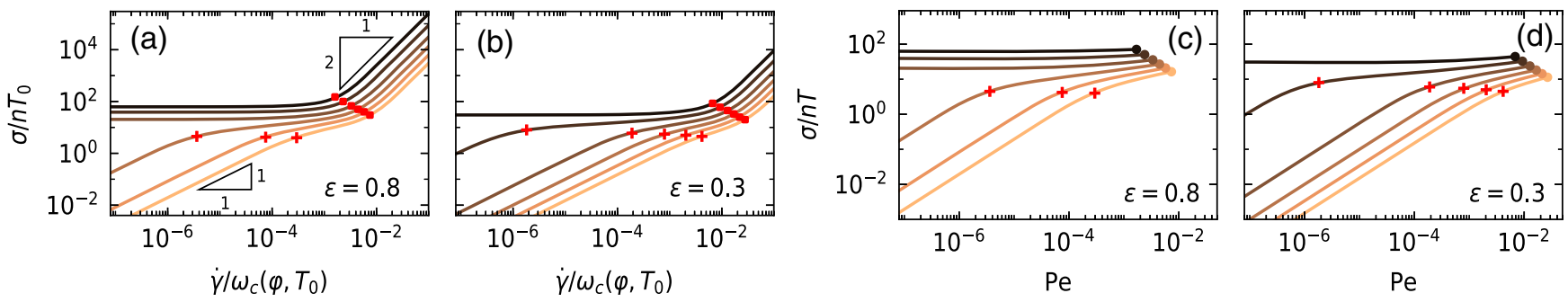

FIG. 2. Flow curves: shear stress $\sigma$ as a function of shear rate $\dot{\gamma}$ (a),(b) or Péclet number Pe (c),(d) for a number of packing fractions and coefficient of restitution $\varepsilon=0.8$ (a),(c), and $\varepsilon=0.3$ (b),(d). The packing fraction increases from bottom $(\varphi=0.48)$ to top $(\varphi=0.58)$. Critical Péclet numbers indicated by symbols $\mathrm{Pe}_{\alpha}(+), \mathrm{Pe}^{*}$ [filled square, omitted in (c),(d) for clarity] and $\mathrm{Pe}_{\infty}$ (filled circle). 

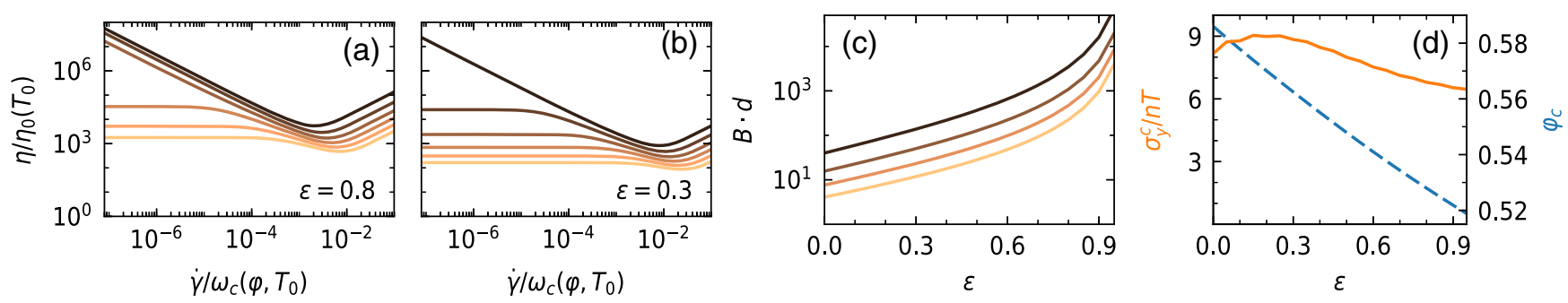

FIG. 3. (a),(b) Viscosity $\eta(\dot{\gamma})$ normalized by the Boltzmann viscosity $\eta_{0}$ as a function of shear rate $\dot{\gamma}$. Same parameters as in Fig. 2. (c) Bagnold coefficient $B$ as a function of coefficient of restitution $\epsilon$ for a few packing fractions from $\varphi=0.48$ (bottom) to $\varphi=0.57$ (top). (d) Yield stress $\sigma_{y}^{c}$ (left axis, solid) at the glass transition packing fraction $\varphi_{c}$ (right axis, dashed, from [44]) as a function of the coefficient of restitution $\varepsilon$.

corresponds to Newtonian rheology and $R>0(R<0)$ to shear thickening (thinning) behavior [47].

Slow shear.-For small shear rates $\mathrm{Pe} \rightarrow 0$ in the linear response regime, the glass transition divides the state space into two qualitatively different phases. For relatively low densities, $\varphi<\varphi_{c}$, GITT provides corrections of order $\varphi^{2}$ to the low-density, Enskog predictions [19] for the viscosity. For densities approaching the glass transition, the divergence of the relaxation time, $\tau_{\alpha}$, results in a divergent viscosity, $\eta\left(\varphi \nearrow \varphi_{c}\right) \propto\left[\varphi_{c}(\varepsilon)-\varphi\right]^{-\gamma(\varepsilon)}$. The critical exponent $\gamma(\varepsilon) \sim 2.5-2.3$ weakly decreases with increasing dissipation [46] and compares well with the experimental value $\gamma \approx 2.35$ [50]. Experimental values for $\varphi_{c}(\varepsilon)$ [51-53] are compatible with our interpretation as a granular glass transition as well. At the glass transition, $\varphi \equiv \varphi_{c}(\varepsilon)$, we find a finite critical yield stress [cf. Fig. 3(d)], $\sigma_{y}^{c}$, in the range $6 n T-9 n T$, compatible with experiments [52], and comparable to theoretical predictions $\left(\sigma_{y}^{c} / n T \sim 6\right.$ [54]) and measurements $\left(\sigma_{y}^{c} / n T \sim 10-15\right.$ [55]) for colloidal suspensions. Thereby placing granular fluids firmly in the realm of soft matter.

Fast shear.-For high Péclet numbers $\mathrm{Pe} \rightarrow \mathrm{Pe}_{\infty}$, Eq. (4) can be used to predict the Bagnold coefficient, $B$ [cf. Fig. 3(c)]. The latter increases with density and elasticity (larger $\varepsilon$ ) as both trends make the temperature more sensitive to changes in the shear rate. In particular, $B$ diverges as $\varepsilon \rightarrow 1$.

As a first quantitative application of GITT, let us compare our predictions to Bagnold's original data [21]. To this end, we extract the Bagnold coefficient, $B$, and the maximal Péclet number, $\mathrm{Pe}_{\infty}$, from his measurements [Fig. 1(c)] [56]. Our kinetic theory proves to recover the qualitative trends of both $B$ and $\mathrm{Pe}_{\infty}$ as a function of packing fraction with no adjustable parameters. Considering that Bagnold's measurements have been shown to leave room for improvement [60] the prediction of GITT compare favorably. Qualitatively the strongly agitated flow curves of Refs. [61,62] agree well with our discussion showing all three regimes. However, note that the packing fraction in the shear zone is unknown and uncontrolled in these experiments in contrast to what is assumed here. More tailored and careful measurements are needed to assess GITT's quantitative accuracy.

Depending on experimental conditions, additional effects may become relevant that go beyond the model considered in this Letter. On Earth (but not, e.g., on the moon [63]) all granular flows are actually two-phase flows of granular particles together with an interstitial fluid (mostly air or water). At a sufficiently low packing fraction, the effective viscosity of the molecular fluid becomes relevant [59]. This will introduce another Newtonian regime at small shear rates that crosses over to Bagnold rheology when the stress induced by the granular particles dominates over the viscous stress of the interstitial fluid. For high shear rates, the Bagnold regime will obviously not extend to $\dot{\gamma} \rightarrow \infty$. At some point typical interparticle forces are so high that interactions can no longer be regarded as hard core. A finite interaction time compared to the shear rate appears as a new timescale and destroys Bagnold scaling [64]. For very high densities, approaching random close packing, the rheology will be dominated by the imminent jamming transition, which is not accounted for in the present model. We prescribe a linear shear profile and a homogeneous constant density and, therefore, necessarily obtain monotonic flow curves. Nonmonotonic, unstable flow curves and the associated discontinuous shear thickening are possible in inhomogeneous systems only $[26,61,62,65]$.

Conclusion.-To summarize, we have discussed that the rich rheology of a granular fluid is controlled by three critical Péclet numbers (Table I): (i) Newtonian rheology prevails for $\mathrm{Pe}<\mathrm{Pe}_{\alpha}$. (ii) For intermediate shear rates, $\mathrm{Pe}_{\alpha}<\mathrm{Pe}<\mathrm{Pe}^{*}$, shear thinning reflects that the shear rate is faster than the intrinsic relaxation rate of the fluid which eventually results in a finite dynamic yield stress above the glass transition density. The latter can be substantially lower than the jamming transition commonly located at random close packing $\varphi_{\text {rcp }} \approx 0.64$ [66]. For low densities, $\mathrm{Pe}_{\alpha} \sim 1$ and at the same time $\mathrm{Pe}^{*} \ll 1$ in the elastic limit $\varepsilon \rightarrow 1$. Under these conditions, $\mathrm{Pe}_{\alpha}>\mathrm{Pe}^{*}$ and the thinning regime vanishes altogether. (iii) For $\mathrm{Pe}^{*}<\mathrm{Pe}<\mathrm{Pe}_{\infty}$ strong shear heating leads to shear thickening behavior which ultimately entails Bagnold scaling as the Péclet 
number approaches its maximum, $\mathrm{Pe} \rightarrow \mathrm{Pe}_{\infty}$. This constitutes yet another shear thickening mechanism different from other mechanisms discussed in the literature, namely clustering [67,68], dilation [69,70], friction [71,72], or steric effects [73]. The fact that $\mathrm{Pe}_{\infty}$ is finite implies that a kinetic theory to predict the Bagnold coefficient $B$ must be applicable at finite shear rates.

To support our arguments and to make them quantitative, we presented a kinetic theory based on the ITT formalism that recovers the phenomenology, covering many orders of magnitude in shear rate and shear stress, or viscosity, respectively. Earlier attempts at formulating ITT for inelastic soft spheres [74] retain no dissipative effects on the same level of approximation. For the inelastic hard sphere fluid considered here, besides the implicit dependence of $\Phi_{\boldsymbol{k}}(t)$ on the coefficient of restitution $\varepsilon$ [46], Eq. (4) also includes an explicit dependence on $\varepsilon$. Thereby we extended quantitative predictions for the transport coefficients of a sheared granular fluid beyond the low-density and low shear rate regime amenable to standard kinetic theories.

The results presented here will be useful as constitutive equations for modeling and simulating large scale granular flows which demand a continuum description. In addition, the Bagnold coefficient is needed for a recent kinetic theory [75]. We also hope that the availability of a kinetic theory for granular shear flow in a range of practically relevant parameters will spur quantitative experiments.

We acknowledge illuminating discussions with Hisao Hayakawa, Koshiro Suzuki, Thomas Voigtmann, and Claus Heussinger. We thank Philip Born for critically reading the manuscript and the DFG for partial funding through FOR 1394 and KR 4867/2-1.

*kranz@thp.uni-koeln.de

[1] C. J. van Westen, T. W. J. van Asch, and R. Soeters, Bull. Eng. Geol. Env. 65, 167 (2006).

[2] P. Höller, Nat. Hazards 43, 81 (2007).

[3] P. Richard, M. Nicodemi, R. Delannay, P. Ribiere, and D. Bideau, Nat. Mater. 4, 121 (2005).

[4] A. Gebhardt, Understanding Additive Manufacturing: Rapid Prototyping-Rapid Tooling-Rapid Manufacturing (Carl Hanser Verlag GmbH Co KG, Munich, 2012).

[5] M. Fateri, A. Gebhardt, S. Thuemmler, and L. Thurn, Phys. Procedia 56, 357 (2014).

[6] P. W. Cleary and M. L. Sawley, Appl. Math. Model. 26, 89 (2002).

[7] H. M. Jaeger and S. R. Nagel, Science 255, 1523 (1992).

[8] L. P. Kadanoff, Rev. Mod. Phys. 71, 435 (1999).

[9] J. Rajchenbach, Adv. Phys. 49, 229 (2000).

[10] M. Ausloos, R. Lambiotte, K. Trojan, Z. Koza, and M. Pękala, Physica (Utrecht) A 357, 337 (2005).

[11] U. M. B. Marconi, A. Puglisi, L. Rondoni, and A. Vulpiani, Phys. Rep. 461, 111 (2008).

[12] A. Prevost, D. A. Egolf, and J. S. Urbach, Phys. Rev. Lett. 89, 084301 (2002).
[13] R. P. Ojha, P.-A. Lemieux, P. K. Dixon, A. J. Liu, and D. J. Durian, Nature (London) 427, 521 (2004).

[14] A. R. Abate and D. J. Durian, Phys. Rev. E 74, 031308 (2006).

[15] S. B. Savage and D. J. Jeffrey, J. Fluid Mech. 110, 255 (1981).

[16] J. T. Jenkins and S. B. Savage, J. Fluid Mech. 130, 187 (1983).

[17] J. J. Brey, J. W. Dufty, C. S. Kim, and A. Santos, Phys. Rev. E 58, 4638 (1998).

[18] N. Sela and I. Goldhirsch, J. Fluid Mech. 361, 41 (1998).

[19] V. Garzó and J. M. Montanero, Physica (Amsterdam) A 313, 336 (2002).

[20] N. V. Brilliantov and T. Pöschel, Kinetic Theory of Granular Gases (Oxford University Press, Oxford, 2004).

[21] R. A. Bagnold, Proc. R. Soc. A 225, 49 (1954).

[22] C. S. Campbell, J. Powder Technol. 162, 208 (2006).

[23] The Physics of Granular Media, edited by H. Hinrichsen and D. E. Wolf (Wiley-VCH, Berlin, 2004).

[24] A. Lemaître, J.-N. Roux, and F. Chevoir, Rheol. Acta 48, 925 (2009).

[25] T. Kawasaki, A. Ikeda, and L. Berthier, Europhys. Lett. 107, 28009 (2014).

[26] E. Brown and H. M. Jaeger, Rep. Prog. Phys. 77, 046602 (2014).

[27] N. Mitarai and H. Nakanishi, Phys. Rev. Lett. 94, 128001 (2005).

[28] K. A. Reddy and V. Kumaran, Phys. Rev. E 76, 061305 (2007).

[29] M. Fuchs and M. E. Cates, Phys. Rev. Lett. 89, 248304 (2002).

[30] J. W. Dufty, Granular Matter 14, 271 (2012).

[31] W. T. Kranz, F. Frahsa, A. Zippelius, M. Fuchs, and M. Sperl, arXiv:1710.04475 [Phys. Rev. Fluids (to be published)].

[32] J. M. Brader, T. Voigtmann, M. Fuchs, R. G. Larson, and M. E. Cates, Proc. Natl. Acad. Sci. U.S.A. 106, 15186 (2009).

[33] M. Fuchs and M. E. Cates, J. Rheol. 53, 957 (2009).

[34] J. M. Brader, T. Voigtmann, M. E. Cates, and M. Fuchs, Phys. Rev. Lett. 98, 058301 (2007).

[35] J. M. Brader, J. Phys. Condens. Matter 22, 363101 (2010).

[36] C. P. Amann, M. Siebenbürger, M. Krüger, F. Weysser, M. Ballauff, and M. Fuchs, J. Rheol. 57, 149 (2013).

[37] A. Nicolas and M. Fuchs, J. Non-Newtonian Fluid Mech. 228, 64 (2016).

[38] S.-H. Chong and B. Kim, Phys. Rev. E 79, 021203 (2009).

[39] K. Suzuki and H. Hayakawa, Phys. Rev. E 87, 012304 (2013)

[40] L. Bocquet, W. Losert, D. Schalk, T. C. Lubensky, and J. P. Gollub, Phys. Rev. E 65, 011307 (2001).

[41] C. Bizon, M. D. Shattuck, J. B. Swift, and H. L. Swinney, Phys. Rev. E 60, 4340 (1999).

[42] In practice, $P_{D}=P_{D}(X, \varphi, \dot{\gamma})$ is not only a function of the control parameters of the driving, $X$ (e.g., flow rate, shaking strength, etc.) but also depends on the state of the system $(\varphi, \dot{\gamma})$. Here, for simplicity, we assume $P_{D}$ is fixed.

[43] A. Lemaître, Phys. Rev. Lett. 89, 064303 (2002).

[44] W. T. Kranz, M. Sperl, and A. Zippelius, Phys. Rev. Lett. 104, 225701 (2010). 
[45] W. T. Kranz and M. Sperl, EPJ Conf. 140, 03064 (2017).

[46] W. T. Kranz, M. Sperl, and A. Zippelius, Phys. Rev. E 87, 022207 (2013).

[47] MCT, using the standard approximations, is known to underestimate the glass transition density for elastic hard spheres $(\varepsilon=1), \varphi_{c}^{\mathrm{MCT}} \approx 0.516$ [48], by about $5.5 \%$ compared to the experimental value $\varphi_{c}^{\text {exp }} \approx 0.57$ [49]. Assuming that this difference remains constant for $\varepsilon<1$, we add a tentatively shifted axis $\hat{\varphi}=\varphi+5.5 \%$ to Fig. 1(a) to facilitate comparison to experiments.

[48] U. Bengtzelius, W. Götze, and A. Sjölander, J. Phys. C 17, 5915 (1984).

[49] W. van Megen, T. C. Mortensen, S. R. Williams, and J. Müller, Phys. Rev. E 58, 6073 (1998).

[50] C. Bonnoit, T. Darnige, E. Clement, and A. Lindner, J. Rheol. 54, 65 (2010).

[51] G. Ovarlez, F. Bertrand, and S. Rodts, J. Rheol. 50, 259 (2006).

[52] E. Brown and H. M. Jaeger, Phys. Rev. Lett. 103, 086001 (2009).

[53] C. Gamonpilas, J. F. Morris, and M. M. Denn, J. Rheol. 60, 289 (2016).

[54] M. Fuchs and M. E. Cates, Faraday Discuss. Chem. Soc. 123, 267 (2003).

[55] G. Petekidis, D. Vlassopoulos, and P. N. Pusey, J. Phys. Condens. Matter 16, S3955 (2004).

[56] See Supplemental Material at http://link.aps.org/ supplemental/10.1103/PhysRevLett.121.148002, including Refs. [57,58], for details and additional analysis of Ref. [59].

[57] M. N. Bannerman, L. Lue, and L. V. Woodcock, J. Chem. Phys. 132, 084507 (2010).

[58] A. Lorenz, C. Tuozzolo, and M. Y. Louge, Exp. Mech. 37, 292 (1997).
[59] A. Fall, A. Lemaître, F. Bertrand, D. Bonn, and G. Ovarlez, Phys. Rev. Lett. 105, 268303 (2010).

[60] M. L. Hunt, R. Zenit, C. S. Campbell, and C. Brennen, J. Fluid Mech. 452, 1 (2002).

[61] J. A. Dijksman, G. H. Wortel, L. T. H. van Dellen, O. Dauchot, and M. van Hecke, Phys. Rev. Lett. 107, 108303 (2011).

[62] G. H. Wortel, J. A. Dijksman, and M. van Hecke, Phys. Rev. E 89, 012202 (2014).

[63] B. P. Kokelaar, R. S. Bahia, K. H. Joy, S. Viroulet, and J. M. N. T. Gray, J. Geophys. Res. Planets 122, 1893 (2017).

[64] D. Vågberg, P. Olsson, and S. Teitel, Phys. Rev. E 95, 012902 (2017).

[65] A. Gnoli, A. Lasanta, A. Sarracino, and A. Puglisi, Sci. Rep. 6 (2016).

[66] C. S. O'Hern, S. A. Langer, A. J. Liu, and S. R. Nagel, Phys. Rev. Lett. 88, 075507 (2002).

[67] J. R. Melrose and R. C. Ball, J. Rheol. 48, 961 (2004).

[68] N. J. Wagner and J. F. Brady, Phys. Today 62, 10, 27 (2009).

[69] E. Brown and H. M. Jaeger, J. Rheol. 56, 875 (2012).

[70] A. Fall, F. Bertrand, G. Ovarlez, and D. Bonn, J. Rheol. 56, 575 (2012).

[71] C. Heussinger, Phys. Rev. E 88, 050201 (2013).

[72] B. M. Guy, M. Hermes, and W. C. K. Poon, Phys. Rev. Lett. 115, 088304 (2015).

[73] F. Picano, W.-P. Breugem, D. Mitra, and L. Brandt, Phys. Rev. Lett. 111, 098302 (2013).

[74] K. Suzuki and H. Hayakawa, in AIP Conference Proceedings (AIP, Melville, 2014), Vol. 1628, p. 457.

[75] V. Kumaran, J. Fluid Mech. 756, 555 (2014). 